\title{
PHOSPHATIDYLCHOLINE CONTAINING LONG CHAIN OMEGA-3 FATTY ACIDS: A TREATMENT ADJUNCT FOR PATIENTS WITH ANOREXIA NERVOSA?
}

\author{
Olivia Patsalos ${ }^{1 *}$, Theodoros Mavrogiannidis ${ }^{1,2^{*}}$, Bethan Dalton ${ }^{1}$, \\ Catherine J. Field ${ }^{3}$ \& Hubertus Himmerich ${ }^{1}$ \\ ${ }^{1}$ Department of Psychological Medicine, Institute of Psychiatry, Psychology \& Neuroscience, King's College London, \\ London, United Kingdom \\ ${ }^{2}$ School of Medicine, Aristotle University of Thessaloniki, Thessaloniki, Greece \\ ${ }^{3}$ Department of Agricultural Food and Nutritional Science, Faculty of Agricultural Life and Environmental Science, \\ University of Alberta, Edmonton, Alberta, Canada
}

*These authors contributed equally to this work

received: 12.12.2019;

revised: 7.2.2020;

accepted: 23.2 .2020

\section{SUMMARY}

Background: Anorexia nervosa (AN) is a serious mental disorder with a high mortality rate and often a chronic course. In contrast to many other common mental disorders, there is no drug therapy approved for AN.

Methods: We performed a narrative literature review to consider whether a choline-containing molecule, such as phosphatidylcholine (PC), with an omega ( $\omega)$-3 long chain polyunsaturated fatty acid (LCPUFA) could be a potential future medicinal treatment for $A N$.

Results: Choline and LCPUFAs have individually shown benefit for mental health. Case series and pilot studies suggest $\omega-3$ LCPUFAs may be effective in eating disorders. However, pharmacodynamic and pharmacokinetic considerations suggest a greater benefit from the combination of both components.

Conclusion: The combination of a choline-containing molecule with an $\omega-3$ LCPUFA may be clinically effective and well tolerated. This idea is supported by the current literature on the role of inflammation, the microbiome, the gut-brain-axis, hormonal, neurotransmitter and intracellular signalling, and on the structure and fluidity of nerve cells membranes in patients with AN.

Key words: omega-3 fatty acids - choline - phosphatidylcholine - cytokines - anorexia nervosa

\section{INTRODUCTION}

Anorexia Nervosa (AN) has one of the highest mortality rates of all psychiatric illnesses (Arcelus et al. 2011). It is defined by restricted calorie intake, malnutrition and body image disturbances (American Psychiatric Association 2013). Given that approximately 20\% of AN patients become chronic sufferers (Steinhausen 2002), there is a need for novel and effective treatment options. We propose that the use of a choline-containing molecule, such as phosphatidylcholine (PC), with an omega ( $\omega)-3$ long chain polyunsaturated fatty acid (LCPUFA) may be a potential future treatment option for AN. Both of these molecules individually have beneficial effects on brain structure and function (Satogami et al. 2017), however a combination of these molecules is not yet commercially available.

\section{POLYUNSATURATED FATTY ACIDS}

Polyunsaturated fatty acids (PUFAs) are long hydrocarbon chains that are essential structural and signalling components of the phospholipids in human cells. Two classes of LCPUFAs, $\omega-3$ fatty acids (FAs) and $\omega-6$ FAs, are particularly important in regulating membrane function (Das 2006) and make up a significant portion of the FAs in the brain (Calder 2016). w3-LCPUFAs cannot be produced by the human body and thus need to be consumed through our diet. The $\omega-3$ FA group includes $\alpha$-linoleic acid (ALA), which is commonly found in plant oils such as flaxseed, soybean and canola oil as well as eicosapentaenoic acid (EPA) and docosahexaenoic acid (DHA), both commonly found in marine oils such as fish, krill and squid oils, and some new algae and single cell sources. Major sources of $\omega-6$ dietary sources include nuts and plant oils and there is a small amount of the $\omega-6$ LCPUFA arachidonic acid (AA) in animal products. The ideal $\omega-6$ to $\omega-3$ ratio in the diet is unknown but is suggested to be somewhere between 2:1 and 4:1 (Simopoulos 2003). However, the estimated dietary ratio in the Western diet is in excess of $15: 1$ due to the high intake of $\omega$-6-rich plant oils (Simopoulos 2003). A low $\omega-3$ LCPUFA status has specifically been found in AN patients, as compared to controls (Caspar-Bauguil et al. 2012).

Choline was recognized as a dietary essential nutrient in 1998 and is required for the synthesis of acetylcholine, sphingomyelin and phosphatidylcholine (PC). It is also a major source of methyl groups (Blusztajn et al. 2017). Neurons have not been found to 
synthesize choline and as such, the brain depends on diet and the endogenous synthesis for a supply of choline (Blusztajn et al. 2017). As choline has important metabolic functions, its deficiency has been hypothesized to contribute to non-alcoholic fatty liver disease, cardiovascular disease, and neurological and psychiatric disorders (Zeisel \& da Costa 2009). De novo synthesis of choline occurs primarily in the liver by methylation of phosphatidylethanolamine (PE) to PC; however, this process does not meet human requirements. Therefore, a dietary source is also needed (Zeisel \& da Costa 2009). Choline in the diet and body is found in a number of forms including the aqueous forms of free unesterified choline, phosphocholine and glycerophosphocholine (GPC) as well as the lipid-soluble molecules PC, lysophosphatidylcholine (lysoPC) and sphingomyelin (Zeisel et al. 2003). In a study of young pregnant women, it was found that PC was the major choline form consumed, contributing $48 \pm 2 \%$ of total intake (Lewis et al. 2014). PC is a choline containing a phospholipid with two FAs attached. Men and postmenopausal women are more susceptible to choline deficiency due to lower estrogen levels, which promotes de novo synthesis (Zeisel \& Caudill 2010). In the healthy population, adolescent girls and young women would not usually be at risk of choline deficiency. However, in AN, disruption of estrogen synthesis together with a low intake of the foods that provide a source of choline in the diet would place these individuals at a similar risk to males. Currently, there is no evidence on which to base diet recommendations for the quantity of choline in the diet of AN patients.

When stimulated, the phospholipids (including PC) in the membrane release $\omega-3$ and $\omega-6$ LCPUFAs to form a number of bioactive eicosanoids (Calder 2015). The $\omega-6$ derived eicosanoids have strong pro-inflammatory effects, whereas $\omega-3$ derived eicosanoids have less inflammatory or anti-inflammatory effects (Calder 2017). The anti-inflammatory effects of $\omega-3$ LCPUFAs may counteract the signalling of pro-inflammatory cytokines which have been suggested to play a pathophysiological role in AN (Dalton et al. 2018) by influencing the action of hormones and neurotransmitters in the three most important neurocircuits of appetite regulation: the selfregulatory, hedonic, and homeostatic systems (Himmerich \& Treasure 2018). Thus, we believe that $\omega-3$ LCPUFA treatment could be a promising candidate for investigation in the context of AN. Administered together with choline, $\omega-3$ LCPUFA treatment might be even more effective.

\section{POTENTIAL BENEFITS OF $\Omega-3$ AND CHOLINE}

$\omega$-3 LCPUFAs exert their anti-inflammatory potential through several interactions. EPA and DHA can partly inhibit components of inflammation including leucocyte chemotaxis, adhesion molecule expression, leucocyte-endothelial adhesive interactions, and the production of eicosanoids (Calder 2015). For these reasons, they have been hypothesized to be potential treatments for a wide range of psychiatric conditions (Prior \& Galduróz 2012). Additionally, $\omega-3$ LCPUFAs can induce the production of inflammation-resolving molecules e.g., resolvins, protectins, and maresins (Calder 2017). $\omega-3$ LCPUFAs can also inhibit the activation of the pro-inflammatory transcription factor, nuclear factor (NF) $-\kappa \mathrm{B}$, through activating the anti-inflammatory transcription factor peroxisome proliferator-activated receptor (PPAR)- $\gamma$, resulting in reduced expression of inflammatory genes. Furthermore, $\omega-3$ LCPUFAs can exert their effects indirectly by counteracting the inflammatory $\omega-6$ FAs (Calder 2017).

A body of research reports an association between gut microbiota dysbiosis and mental health (Iannone et al. 2019). $\omega$-3 LCPUFAs have been shown to modulate the intestinal environment by altering the gut microbiota composition, leading to an increase in the population of bacterial taxa that promote eubiosis, rather than dysbiosis and inflammation (see Costantini et al. 2017 for a review). Furthermore, through their effects on the gut microbiota, $\omega$-3 LCPUFAs have been found to influence the gut-brain axis (Sampson \& Mazmanian, 2015), which plays an important role in mental health (Scriven et al. 2018). Both a lower $\omega$-3 LCPUFA status (Caspar-Bauguil et al. 2012) and altered microbiome diversity (Seitz et al. 2019) has been implicated in AN.

Further to their anti-inflammatory potential, $\omega-3$ LCPUFAs may be of relevance to AN patients through their other functions (see Shih et al. 2017 for a review). For example, Yehuda \& Rabinovitz (2016) have postulated that the beneficial effects that $\omega$-3 LCPUFAs have on AN patients may be due to their ability to stabilise the neuronal membrane fluidity index. It was recently suggested that the differential postprandial $\omega 6$-derived eicosanoid shift that has been reported in those with AN might contribute to the food aversion that is typical of these patients (Shih et al. 2017). In support of this, LCPUFAs have been shown to increase appetite (Damsbo-Svendsen et al. 2013), enhance endocannabinoidmediated neuronal functions (Lafourcade et al. 2011), and restore the disturbed blood-brain barrier functions (Yehuda et al. 2005).

Supplementation with choline has been studied in a number of neurological diseases in adults, including age-related memory and cognitive decline, Alzheimer's disease, epilepsy, and inherited conditions, such as Down and Rett syndromes, with promising results (for review see Blusztajn et al. 2017). These studies supplemented choline as a salt (free choline). There is, however, a lack of studies using PC as a treatment for brain disorders. A medication combining choline and $\omega-3$ LCPUFAs is not yet commercially produced. However, hypothetically, PC could be synthesized to contain an $\omega-3$ LCPUFA in at least one of the FA positions. It would be favourable to place this $\omega-3$ LCPUFA in the sn-2 position as this has the potential to be absorbed and 
transported across the blood brain barrier (Hachem et al. 2016). PC and $\omega-3$ LCPUFAs have very few side effects. The most commonly reported are gastrointestinal disturbances, such as nausea, and a fishy aftertaste (when fish is the source of the $\omega-3$ LCPUFAs). Given their mild nature, these side effects rarely influence patient adherence (Freeman et al. 2010, Akhondzadeh et al. 2013). Indeed, in AN samples, w-3 LCPUFA supplementation has generally been reported to be acceptable and well-tolerated (Manos et al. 2018, Woo et al. 2017, Ayton et al. 2004).

\section{CLINICAL RESEARCH IN AN}

$\omega$-3 LCPUFA supplementation in the context of AN is in its infancy and has therefore, only been examined in a few studies in AN specifically. In a randomized controlled trial (RCT) investigating EPA supplementation $(480 \mathrm{ml}, 620 \mathrm{kcal}, 32 \mathrm{~g}$ protein $\pm 2.2 \mathrm{~g} \mathrm{EPA})$ in cancer cachexia, a wasting syndrome that leads to weight loss like in AN, the use of EPA was found to ameliorate the loss of skeletal muscle mass exhibited by these patients (Fearon et al. 2003). In people with AN specifically, we identified one case series of seven adolescent patients supplemented with EPA (1 g/day EEPA). The authors reported an increase in body weight and an improvement in anorexia psychopathology and depressive symptoms (Ayton et al. 2004). However, not all studies have been as promising. For example, in an $\mathrm{RCT}$ in adolescent girls with $\mathrm{AN}(\mathrm{n}=53)$, there were no significant differences between supplementation with PUFAs $(558 \mathrm{mg}$ of eicosapentaenoic acid, $174 \mathrm{mg}$ of docosahexaenoic acid and $60 \mathrm{mg}$ of gamma linolenic acid) for 10 weeks during inpatient treatment, compared to placebo, in depressive and obsessive and compulsive symptoms (Pirog-Balcerzak et al. 2016). However, weight and ED symptom outcomes were not reported. Furthermore, Manos et al. (2018) conducted a small pilot RCT $(n=24)$ in which adolescent girls with AN were supplemented with either $2,120 \mathrm{mg}$ eicosapentaenoic acid/ $600 \mathrm{mg}$ docosahexaenoic acid or placebo for 12 weeks, finding no difference in weight, eating disorder symptoms, or depression between the groups at the end of the intervention.

A high percentage of $\mathrm{AN}$ patients present with psychiatric co-morbidities, most notably depression (Ulfvebrand et al. 2015). Interestingly, Swenne et al. (Swenne et al. 2011) reported lower amounts of EPA and DHA in the erythrocyte membranes of adolescents presenting with EDs and depression, as compared to those presenting with only AN. In line with this, studies examining the effects of LCPUFAs on depressive symptoms (in patients both with and without $\mathrm{AN}$ ) found that supplementation with EPA can decrease both inflammation and depressive symptoms (Rapaport et al. 2016, Peet \& Horrobin 2002, Grosso et al. 2014, Jacka et al. 2013). Furthermore, adjunctive treatment with $\omega-3$ LCPUFAs was found to augment the therapeutic efficacy of fluoxetine (Jazayeri et al. 2008) and citalopram (Gertsik et al. 2012).

To our knowledge, choline or PC supplementation has not been studied in AN. There has been one animal study that suggested that dietary choline could stabilize brain function in rats with induced anorexia (Wöckel et al. 2007). Despite the limited evidence in AN, there have been a number of small clinical trials or cohort studies with patients with various weight-related and neurological disorders, such as obesity (see Edwards et al. 2019).

\section{CONCLUSION AND FUTURE PERSPECTIVES}

The use of a combination of choline and $\omega-3$ LCPUFAs for the treatment of $\mathrm{AN}$ is a novel idea. Given the promising results for the use of either $\omega-3$ LCPUFAs or choline in related illnesses, there is a clinical rationale for examining the combination of both nutrients as a treatment adjunct in patients with AN. These naturally derived, well-tolerated and low-cost agents could be a potential therapeutic tool for the treatment of $\mathrm{AN}$, for which there are currently no approved pharmacological treatments available (Himmerich \& Treasure 2018). We recommend the investigation of a novel PC molecule containing $\omega-3$ LCPUFAs within RCTs, whose primary outcome measures should include weight changes, body composition changes (e.g., muscle mass, fat mass, etc), dietary intake, inflammatory marker changes, and general and AN-specific psychopathology as captured by the Eating Disorder Examination Questionnaire (EDE-Q, Fairburn \& Beglin 2008). Additionally, recent genome-wide-association studies (GWAS) have shown genetic correlations of AN with schizophrenia, obsessive-compulsive disorder and depression (CrossDisorder Group of the Psychiatric Genomics Consortium 2019, Himmerich et al. 2019). These correlations mirror the shared clinical symptoms between AN and these psychiatric disorders, such as overvalued ideas (body image disturbance), pseudo-hallucinations (the so-called anorexic voice), rituals, obsessions, and depressed mood (Himmerich \& Treasure 2018). Thus, we would further recommend the use of questionnaires covering the symptoms of genetically- and clinicallyrelated disorders such as the Brief Psychiatric Rating Scale (BPRS, Overall \& Gorham 1962), the Yale-Brown Obsessive Compulsive Scale (Y-BOCS, Goodman et al. 1989) and the Depression Anxiety Stress Scales (DASS, Lovibond \& Lovibond 1995).

We also suggest the involvement of patients in the design of these studies, along with the inclusion of patient-related outcomes and measures of experience and quality of life. A recent clinical audit revealed that patients want help with anxiety, concentration, sleep problems and anorexic thoughts (Dessain et al. 2019). Therefore, it would be of benefit to patients if these concerns were assessed in future RCTs. Such carefully 
designed RCTs could provide an opportunity to explore the potential efficacy of choline and $\omega-3$ LCPUFAs as a treatment modality in AN.

Relatedly, it would be of interest to explore the effects of these nutrients in the broader context of psychiatric disorders that frequently co-occur and share genetic risk factors with AN (Himmerich \& Treasure 2018; Himmerich et al. 2019). For example, depression, anxiety, and PTSD, which are also associated with inflammation and brain structure abnormalities, may also benefit from potential treatments that combine choline and $\omega-3$ LCPUFAs.

\section{Acknowledgements:}

\section{Funding statement:}

Theodoros Mavrogiannidis received Erasmus Plus funding via Aristotle University of Thessaloniki for a studentship at King's College London (KCL). Bethan Dalton is supported by a studentship awarded by the Department of Psychological Medicine, KCL and the Institute of Psychiatry, Psychology and Neuroscience (IoPPN), KCL. Hubertus Himmerich has received salary support from the NIHR BRC at the South London and Maudsley NHS Foundation Trust (SLaM) and $\mathrm{KCL}$.

\section{Conflict of interest: None to declare.}

\section{Contribution of individual authors:}

Catherine J. Field \& Hubertus Himmerich had the main scientific ideas which are the intellectual basis for this manuscript.

The first draft was written by Theodoros Mavrogiannidis.

Olivia Patsalos \& Bethan Dalton revised this first draft substantially.

All authors discussed the manuscript and approved the final submitted version.

\section{References}

1. Akhondzadeh S, Gerbarg PL, Brown RP: Nutrients for Prevention and Treatment of Mental Health Disorders. Psychiatr Clin North Am 2013; 36:25-36

2. American Psychiatric Association: Diagnostic and statistical manual of mental disorders (5th ed.). APA Press, Washington DC, 2013

3. Arcelus J, Mitchell AJ, Wales J, Nielsen S: Mortality Rates in Patients With Anorexia Nervosa and Other Eating Disorders. Arch Gen Psychiatry 2011; 68:724

4. Ayton AK, Azaz A, Horrobin DF: A pilot open case series of Ethyl-EPA supplementation in the treatment of anorexia nervosa. Prostaglandins, Leukot Essent Fat Acids 2004; 71:205-209

5. Blusztajn JK, Slack BE, Mellott TJ: Neuroprotective Actions of Dietary Choline. Nutrients 2017; 9:815

6. Calder PC: Docosahexaenoic Acid. Ann Nutr Metab 2016; 69:8-21
7. Calder PC: Marine omega-3 fatty acids and inflammatory processes: Effects, mechanisms and clinical relevance. Biochim Biophys Acta - Mol Cell Biol Lipids 2015; 1851:469-484

8. Calder PC: Omega-3 fatty acids and inflammatory processes: from molecules to man. Biochem Soc Trans 2017; 45:1105-1115

9. Caspar-Bauguil S, Montastier E, Galinon F, FrischBenarous D, Salvayre R, Ritz P: Anorexia nervosa patients display a deficit in membrane long chain polyunsaturated fatty acids. Clin Nutr 2012; 31:386-390

10. Costantini L, Molinari R, Farinon B, Merendino N: Impact of Omega-3 Fatty Acids on the Gut Microbiota. Int $J$ Mol Sci 2017; 18

11. Cross-Disorder Group of the Psychiatric Genomics Consortium: Genomic Relationships, Novel Loci, Pleiotropic Mechanisms across Eight Psychiatric Disorders. Cell 2019; 179:1469-1482

12. Dalton B, Bartholdy S, Robinson L, Solmi M, Ibrahim MAA, Breen $G$ et al.: A meta-analysis of cytokine concentrations in eating disorders. J Psychiatr Res 2018; 103:252-264

13. Damsbo-Svendsen S, Rønsholdt MD, Lauritzen L: Fish oil-supplementation increases appetite in healthy adults. A randomized controlled cross-over trial. Appetite 2013; 66:62-66

14. Das UN: Essential fatty acids: biochemistry, physiology and pathology. Biotechnol J 2006; 1:420-439

15. Dessain A, Bentley J, Treasure J, Schmidt U, Himmerich H: Patients' and Carers' Perspectives of Psychopharmacological Interventions Targeting Anorexia Nervosa Symptoms. In: Himmerich H, Jauregui Lobera I (Eds): Anorexia and Bulimia Nervosa. IntechOpen: London 2019, pp 103-122

16. Edwards CG, Walk AM, Cannavale CN, Flemming IR, Thompson $S$ V., Reeser GR et al.: Dietary choline is related to neural efficiency during a selective attention task among middle-aged adults with overweight and obesity. Nutr Neurosci 2019; 1-10

17. Fairburn CG Beglin SJ: Eating Questionnaire. Cogn Behav Ther Eat Disord 2008; 12-14

18. Fearon KCH, Von Meyenfeldt MF, Moses AGW, Van Geenen R, Roy A, Gouma DJ et al.: Effect of a protein and energy dense N-3 fatty acid enriched oral supplement on loss of weight and lean tissue in cancer cachexia: a randomised double blind trial. Gut 2003; 52:1479-86

19. Freeman MP, Fava M, Lake J, Trivedi MH, Wisner KL, Mischoulon D: Complementary and Alternative Medicine in Major Depressive Disorder. J Clin Psychiatry 2010; 71:669-681

20. Gertsik L, Poland RE, Bresee C, Rapaport MH: Omega-3 Fatty Acid Augmentation of Citalopram Treatment for Patients With Major Depressive Disorder. J Clin Psychopharmacol 2012; 32:61-64

21. Goodman WK, Price LH, Rasmussen SA, Mazure C, Fleischmann RL, Hill CL et al.: The Yale-Brown Obsessive Compulsive Scale: I. Development, Use, and Reliability. Arch Gen Psychiatry 1989; 46:1006-1011

22. Grosso G, Galvano F, Marventano S, Malaguarnera M, Bucolo C, Drago F et al.: Omega-3 Fatty Acids and Depression: Scientific Evidence and Biological Mechanisms. Oxid Med Cell Longev 2014; 2014:1-16 
23. Hachem M, Géloën A, Lo Van A, Foumaux B, Fenart L, Gosselet F et al.: Efficient Docosahexaenoic Acid Uptake by the Brain from a Structured Phospholipid. Mol Neurobiol 2016; 53:3205-3215

24. Himmerich H, Bentley J, Kan C, Treasure J: Genetic risk factors for eating disorders: an update and insights into pathophysiology. Ther Adv Psychopharmacol 2019; 9:204512531881473

25. Himmerich H, Treasure J: Psychopharmacological advances in eating disorders. Expert Rev Clin Pharmacol 2018, 11:95-108

26. Iannone LF, Preda A, Blottière HM, Clarke G, Albani D, Belcastro V et al.: Microbiota-gut brain axis involvement in neuropsychiatric disorders. Expert Rev Neurother 2019; 1-14

27. Jacka FN, Pasco JA, Williams LJ, Meyer BJ, Digger R, Berk M: Dietary intake of fish and PUFA, and clinical depressive and anxiety disorders in women. $\mathrm{Br} J$ Nutr 2013; 109:2059-2066

28. Jazayeri S, Tehrani-Doost M, Keshavarz SA, Hosseini M, Djazayery A, Amini $H$ et al.: Comparison of Therapeutic Effects of Omega-3 Fatty Acid Eicosapentaenoic Acid and Fluoxetine, Separately and in Combination, in Major Depressive Disorder. Aust New Zeal J Psychiatry 2008; 42:192-198

29. Lafourcade M, Larrieu T, Mato S, Duffaud A, Sepers M, Matias I et al.: Nutritional omega-3 deficiency abolishes endocannabinoid-mediated neuronal functions. Nat Neurosci 2011; 14:345-350

30. Lewis ED, Subhan FB, Bell RC, McCargar LJ, Curtis JM, Jacobs RL et al.: Estimation of choline intake from $24 \mathrm{~h}$ dietary intake recalls and contribution of egg and milk consumption to intake among pregnant and lactating women in Alberta. Br J Nutr 2014; 112:112-121

31. Lovibond SH, Lovibond PF. Manual for the depression anxiety stress scales. Psychology Foundation of Australia, Sydney, 1995

32. Manos BE, Bravender TD, Harrison TM, Lange HLH, Cottrill CB, Abdel-Rasoul $M$ et al.: A pilot randomized controlled trial of omega-3 fatty acid supplementation for the treatment of anxiety in adolescents with anorexia nervosa. Int J Eat Disord 2018; 51:1367-1372

33. Overall JE, Gorham DR: The Brief Psychiatric Rating Scale. Psychol Rep 1962; 10:799-812

34. Peet M, Horrobin DF: A dose-ranging study of the effects of ethyl-eicosapentaenoate in patients with ongoing depression despite apparently adequate treatment with standard drugs. Arch Gen Psychiatry 2002; 59:913-9

35. Pirog-Balcerzak A, Bażyńska A, Bragoszewska J, Niwiński P, Popek L, Remberk B et al.: Polyunsaturated omega-3 fatty acids are not effective for depressive and compulsive symptoms in adolescent girls with anorexia nervosa. Eur Neuropsychopharmacol 2016; 26:729-730

36. Prior PL, Galduróz JCF: (N-3) Fatty Acids: Molecular Role and Clinical Uses in Psychiatric Disorders. Adv Nutr 2012; 3:257-265

37. Rapaport MH, Nierenberg AA, Schettler PJ, Kinkead B, Cardoos $A$, Walker $R$ et al.: Inflammation as a predictive biomarker for response to omega-3 fatty acids in major depressive disorder: a proof-of-concept study. Mol Psychiatry 2016; 21:71-79

38. Sampson TR, Mazmanian SK: Control of Brain Development, Function, and Behavior by the Microbiome. Cell Host Microbe 2015; 17:565-576

39. Satogami K, Tseng P-T, Su K-P, Takahashi S, Ukai S, Li $D-J$ et al.: Relationship between polyunsaturated fatty acid and eating disorders: Systematic review and metaanalysis. Prostaglandins Leukot Essent Fatty Acids 2019; 142:11-19

40. Scriven M, Dinan T, Cryan J, Wall M: Neuropsychiatric Disorders: Influence of Gut Microbe to Brain Signalling. Diseases 2018; 6:78

41. Seitz J, Trinh S, Herpertz-Dahlmann B: The Microbiome and Eating Disorders. Psychiatr Clin North Am 2019; 42:93-103

42. Shih PB, Morisseau C, Le T, Woodside B, German JB: Personalized polyunsaturated fatty acids as a potential adjunctive treatment for anorexia nervosa. Prostaglandins Other Lipid Mediat 2017; 133:11-19

43. Simopoulos AP: Importance of the ratio of omega6/omega-3 essential fatty acids: evolutionary aspects. World Rev Nutr Diet 2003; 92:1-22

44. Steinhausen H-C: The Outcome of Anorexia Nervosa in the 20th Century. Am J Psychiatry 2002; 159:1284-1293

45. Swenne I, Rosling A, Tengblad S, Vessby B: Omega-3 polyunsaturated essential fatty acids are associated with depression in adolescents with eating disorders and weight loss. Acta Paediatr 2011; 100:1610-1615

46. Ulfvebrand S, Birgegård A, Norring C, Högdahl L, von Hausswolff-Juhlin Y: Psychiatric comorbidity in women and men with eating disorders results from a large clinical database. Psychiatry Res 2015; 230:294-299

47. Wöckel L, Bertsch T, Koch S, Achtnichts L, Holtmann M, Gretz $N$ et al.: The importance of choline and different serum parameters for the course of the anorexia nervosa. Fortschr Neurol Psychiatr 2007; 75:402-12

48. Woo J, Couturier J, Pindiprolu B, Picard L, Maertens C, Leclerc A et al.: Acceptability and tolerability of omega-3 fatty acids as adjunctive treatment for children and adolescents with eating disorders. Eat Disord 2017; 25:114-121

49. Yehuda S, Rabinovitz S: The Role of Essential Fatty Acids in Anorexia Nervosa and Obesity. Crit Rev Food Sci Nutr 2016; 56:2021-2035

50. Yehuda S, Rabinovitz S, Mostofsky DI: Essential fatty acids and the brain: From infancy to aging. Neurobiol Aging 2005; 26:98-102

51. Zeisel SH: Nutritional importance of choline for brain development. J Am Coll Nutr 2004; 23:621-626

52. Zeisel SH, Caudill MA: Choline. Adv Nutr 2010; 1:46-48

53. Zeisel SH, da Costa $K-A$ : Choline: an essential nutrient for public health. Nutr Rev 2009; 67:615-623

54. Zeisel SH, Mar M-H, Howe JC, Holden JM: Concentrations of Choline-Containing Compounds and Betaine in Common Foods. J Nutr 2003; 133:1302-1307

Correspondence:

Hubertus Himmerich, MD

Department of Psychological Medicine, King's College London

103 Denmark Hill, London SE5 8AF, UK

E-mail: hubertus.himmerich@kcl.ac.uk 